\title{
Introduction to the Network Analysis of Digital and Social Media Minitrack
}

\author{
Devan Rosen \\ Roy H. Park School of Communications \\ Ithaca College \\ Email: drosen@ithaca.edu
}

\author{
George A. Barnett \\ Department of Communication \\ University of California, Davis \\ Email: gbarnett@davis.edu
}

One of the defining characteristics of the information age is the emergence of a new dominant paradigm [1]. During the industrial age the prevailing perspective focused individual components (people or more complex entities), which were defined by their attributes. Their specific behavior was predicted from these traits. The new emerging paradigm, known as the network perspective, is based on the notion that information flows or other relationships exist among the components that constitute the system in which they are embedded and that the behavior of an individual component is determined by these relations. Also, emerging from these relationships is the system's overall structure. System processes may be represented as changes in these relations. The network perspective has been applied throughout the social and physical sciences, as well as redefined our understanding of ecological systems and the role of humanity in our world. Network science has progressed in parallel with the development of social and digital media, computers, the Internet and other information systems [2] [3]. This has provided social and communication network scientists with precise representations of information flows and advanced the state of the science. Additionally, the increased theoretical understanding and analytic representation of computer and information systems provides scientists and engineers with a greater sense of how people and organizations utilize technology to manage resources embedded in their social and digital networks, hence the emergence of a new paradigm for the information age. The four papers selected to represent the Network Analysis of Digital and Social Media are representative of this new perspective.

Natural systems have four essential properties. One is wholeness, where the focus is on the whole system rather than its individual parts. The second property is self-regulation. The components' interactions constrain each other's behavior, thus facilitating the coordination of their behavior to achieve system goals and ensure relative stability. Third is adaptation. Systems evolve over time as the relations among the components change in response to environmental dynamics. Also, some components join the network, while others leave the system. Fourth, systems are composed of individual parts, which together form subsystems, which are themselves embedded in larger supra-systems. This final property is known as hierarchical embeddedness.

Network analysis is an operationalization of the systems perspective. The complex social networks that people create and manage are dynamic, multi-modal, and increasingly mediated by social and digital media. Further, to understand these systems one must take a holistic perspective where the activities of individual components can only be explained by considering the relations among the various parts and larger systems in which they are embedded. The papers in this minitrack are examples of the researchers attempts to digital and social networks

"Applications of Cohesive Subgraph Detection Algorithms to Analyzing Socio-Technical Networks" by Dan Suthers illustrates applications of community detection algorithms (CSDA) to graphs modeling interaction and mediated association. It reviews some leading candidate algorithms (InfoMap, link communities, the Louvain method, and weakly connected components), and evaluates them with respect to how useful they have been in analyzing a large dataset derived from a network of educators known as Tapped In. This practitioner-oriented evaluation is a complement to more formal benchmark based studies common in the literature.

Yu Xu , Yusi Liu and Li Qi describe Wikipedia as a website where independent contributors discuss and negotiate the meanings of past events collaboratively in their paper, "Examining Collective Memory Building in Wikipedia: A Multilevel Network Approach.” They examine how interconnections between high-impact events lead to the different patterns of collective memory. The results show that the presence of a direct network tie between two events is related to a smaller difference in the patterns of collective memory among Wikipedia users. In addition, a high degree of structural equivalence between two events makes them 
similar in collective memory. Their findings reveal that network effects are not confined to the local substructure but can extend to the global level.

In "Cross-National Proximity in Online Social Network and Protest Diffusion: An Event History Analysis of Arab Spring", K. Hazel Kwon and Jeff Hemsley examine the role of online social network proximity in cross-national diffusion of offline protests. Drawing upon a network diffusion model, they operationalizes social network proximity-based protest exposure, using the international Facebook friendship data. Onsite protests during Arab Spring for 2011 were examined using event history modeling. Their findings offer evidence of an contemporaneous online network exposure effect on cross-national diffusion of protests.

The final paper, by Chung-hong Chan and King-wa Fu, entitled, "Facebook Pages' Strength of Ties and Segregation of Engaged Users: An Examination of the Hierarchical Model of Cyberbalkanization", examines the macro-micro linkage between a set of Facebook public pages' tie strength and the segregation of their engaged users. First, a hierarchical model of cyberbalkanization of social media was proposed. Next, a Facebook page-sharing network was created. The network's weighted edge represents the strength of ties between pages. Then, they estimated each page's crowd of unique engaged users, including those who have "liked" and/or made comment to a post of the page and computed the segregation of engaged users of a pair of pages. Using network regression, they found that the strength of ties between Facebook pages was associated with user segregation.

\section{References}

[1] Kuhn, T.S. (1962). The Structure of Scientific Revolutions. Chicago: University of Chicago Press.

[2] Barnett, G.A. (Ed.) Encyclopedia of Social Networks, Thousand Oaks, CA: Sage, 2011.

[3] Rosen, D., Barnett, G.A., \& Kim, J.H., Social networks and online environments: When science and practice coevolve, Social Networks and Mining, 1, 27-42. 2011. 